\title{
Inhibitory motor control in old age: evidence for de-automatization?
}

\section{Elizabeth Ann Maylor*, Kulbir Singh Birak and Friederike Schlaghecken}

Department of Psychology, University of Warwick, Coventry, UK

\section{Edited by:}

Shulan Hsieh, National Cheng Kung

University, Taiwan

\section{Reviewed by:}

Rolf Verleger, Universität zu Lübeck, Germany

Fabienne Collette, Université de Liège, Belgium

John Richard Jennings, University of Pittsburgh, USA

\section{${ }^{*}$ Correspondence:}

Elizabeth Ann Maylor, Department of Psychology, University of Warwick,

Coventry CV4 7AL, UK.

e-mail:e.a.maylor@warwick.ac.uk
To examine age-related effects on high-level consciously controlled and low-level automatically controlled inhibitory processes, the Simon task was combined with the masked prime task in a hybrid procedure. Young and older adults responded to the identity of targets (left/right key-press to left-/right-pointing arrows) that appeared on the left/right of the screen and were preceded by left-/right-pointing backward-masked arrow primes at fixation. Responses were faster and more accurate when the target was congruent with its location than incongruent (Simon effect), and when the target was incompatible with the prime than compatible (negative compatibility effect; NCE). The Simon effect was disproportionately larger, and the NCE disproportionately delayed, in older adults compared to young adults, indicating both high- and low-level inhibitory control deficits with aging. Moreover, the two effects were additive in young adults, but interactive in older adults, providing support for the dedifferentiation hypothesis of aging. Specifically, older adults' prime-related inhibitory control appeared improved on incongruent relative to congruent trials, suggesting that impaired automatic control was substituted by high-level, non-automatic processes.

Keywords: aging, inhibition, cognitive control, masked priming, negative compatibility effect, Simon effect, dedifferentiation

\section{INTRODUCTION}

It has long been supposed that old age is associated with a decrement in the ability to ignore irrelevant information (Rabbitt, 1965), a notion developed by Hasher and Zacks (1988) into the reduced inhibition hypothesis of aging. Supportive evidence comes from several domains, including memory, attention, and motor control (see Hasher et al., 1999; Zacks et al., 2000), and is consistent with neurophysiological data showing marked age-related deterioration of the frontal lobes, traditionally associated with executive control functions (West, 1996; Raz, 2000; Dennis and Cabeza, 2008). However, there have also been critiques of the notion of reduced inhibition as a universal aging phenomenon (e.g., Burke, 1997; McDowd, 1997), resulting in several refinements to the general hypothesis [e.g., see Guerreiro et al's (2010) proposal that agerelated distractibility is modality dependent].

Of particular relevance here are observations of spared inhibitory control in old age (see Kramer et al., 1994; Maylor et al., 2005, for examples) that suggest a distinction should be drawn between different levels of control over inhibitory processes. According to this account, highlevel, consciously controlled inhibitory processes tend to be impaired by normal aging, whereas low-level, more automatically controlled inhibitory processes may be spared (see Andres et al., 2008; Collette et al., 2009). In addition, there is recent evidence of ability dedifferentiation with aging, that is, a stronger coupling between different cognitive processes in old age (Baltes and Lindenberger, 1997; Li et al., 2004). For example, Germain and Collette (2008) found that whereas inhibitory processes in the perceptual and motor domains were additive in young adults, they significantly interacted in older adults.

In the present study, high and low levels of inhibitory control were investigated together in a hybrid experiment combining the Simon spatial congruency task ( $\mathrm{Lu}$ and Proctor, 1995) with the masked prime paradigm (Eimer and Schlaghecken, 1998). A previous study with young adults using this hybrid task (Schlaghecken, Refaat, and Maylor, in press) demonstrated that high- and lowlevel inhibitory control processes act independently of each other, consistent with their putative frontal vs. non-frontal localizations. The main questions of interest here were (1) would older adults show reduced inhibition at one or both levels of control, and (2) would the effects interact, as predicted by the dedifferentiation hypothesis of aging (cf. Li et al., 2004; Germain and Collette, 2008)?

In a typical Simon task (see Proctor and Vu, 2006, for an overview), participants have to give a spatial response to the (arbitrarily assigned) task-relevant feature of a target stimulus (e.g., give a left- or right-hand key-press depending on the target's shape), while refraining from responding to its task-irrelevant location (e.g., appearing to the left or right of fixation). When task-relevant and task-irrelevant information is associated with different responses, inhibitory control is required to resolve the conflict between the voluntarily activated (relevant) response and the automatically activated (irrelevant) response. Such conflict resolution is associated with neural activity primarily in the dorsolateral prefrontal cortex and anterior cingulate cortex (e.g., Peterson et al., 2002; Liu et al., 2004). Simon effects, as measured by response time (RT) differences between congruent and incongruent trials (i.e., where the task-irrelevant stimulus location matches vs. mismatches the required spatial response), have almost always been found to be significantly larger in older than in young adults, with few age differences in error rates (Van der Lubbe and Verleger, 2002; Bialystok et al., 2004; Proctor et al., 2005; Castel et al., 2007; Germain and Collette, 2008; Juncos-Rabadán et al., 2008; Duchek et al., 2009; Kubo-Kawai and Kawai, 2010; Tse et al., 2010). Importantly, these age-related increases in the Simon effect remained significant 
after various measures were taken to control for general slowing in old age (Cerella, 1990; Myerson et al., 1990; Salthouse, 1996), indicating disproportionate age-related decrements. In rare cases where Simon effects were equivalent in young and older adults, the irrelevant location information was conveyed by an accessory stimulus (Proctor et al., 2005, accessory conditions; Simon and Pouraghabagher, 1978), or responses frequently had to be withheld (Kubo-Kawai and Kawai, 2010, go/no-go condition).

In the masked prime paradigm (e.g., Eimer and Schlaghecken, 1998), participants give a speeded response to a simple visual target (e.g., a left-hand response to an arrow pointing to the left). Each target is preceded by a prime stimulus, which is associated with either the same response as the subsequent target (compatible trial), or with a different response (incompatible trial). Primes are presented very briefly (e.g., 17 or $33 \mathrm{~ms}$ ) and backward-masked so that they are unlikely to be perceived consciously, as evidenced by participants' inability to identify primes with more than chance accuracy (Schlaghecken and Eimer, 1997; Eimer and Schlaghecken, 1998, 2002). Responses are faster and more accurate on compatible than on incompatible trials (positive compatibility effect, PCE) when prime and target are presented in immediate succession, but with a short delay between prime offset/mask onset and target onset ( $\sim 150 \mathrm{~ms})$ the reverse is true (negative compatibility effect; NCE). This NCE is assumed to reflect an inhibition process that suppresses the initially activated motor response (e.g., Schlaghecken and Eimer, 2002, 2006; Schlaghecken et al., 2007; Sumner, 2007; Boy et al., 2008; Jaśkowski, 2008b). Schlaghecken et al. (2009) demonstrated that this inhibition occurs at the level of motor response representations. Some have argued that the inhibitory process is triggered by the (conscious) perception of the mask (e.g., Jaśkowski, 2008b), implying that it reflects a top-down control process. However, evidence from both hemodynamic (Aron et al., 2003) and patient studies (Sumner et al., 2007) suggests that this inhibition relies on subcortical and supplementary motor cortical areas, indicating that it differs from the frontally controlled (high-level) inhibition triggered by consciously perceived stimuli as in the Simon task. In line with this, Schlaghecken and Sisman (2006) found that children (7- and 12-year olds) produced NCEs that were indistinguishable from those of young adults, even though they showed considerably less overt response control, corresponding to their as yet immature frontal lobes.

Data from older adults with the masked prime paradigm have been mixed. Whereas Sumner et al. (2007) observed NCEs in older adults (though note there were no young adults in their study for comparison), Schlaghecken and Maylor (2005) failed to find any NCEs in older adults ${ }^{1}$. In the latter case, this was despite evidence of significant PCEs for targets immediately following primes (i.e., there was no age-related decrement in initial activation), and despite extending the prime/mask-target interval to allow for the possibility of a delayed NCE because of general slowing. In a recent larger-scale study, using a fine-grained approach to each individual's time course of effects, Schlaghecken, Birak, and Maylor (in press) demonstrated that the majority of older adults do, in fact, produce NCEs of the same magnitude as those shown by young adults; however, these occur at onset latencies that are disproportionately

${ }^{1}$ For some evidence of delayed NCEs in middle-aged adults, see Seiss and Praamstra (2004, 2006). delayed (i.e., significantly greater than general slowing) and more variable than those of young adults. Thus contrary to notions of spared low-level inhibitory control (Andres et al., 2008; Collette et al., 2009), there is evidence for a disproportionate impairment of low-level inhibitory motor control in old age.

Schlaghecken, Refaat, and Maylor (in press) combined Simon and masked prime tasks in a hybrid procedure to explore the relationship between high-level and low-level response inhibition in young adults. Participants had to respond to the direction of a left- or right-pointing target arrow, ignoring both the target's location (to the left or right of fixation) and a preceding (by $150 \mathrm{~ms}$ ) masked prime arrow at fixation. Both Simon effects and NCEs were observed, but, importantly, no interaction between them. Analyses of serial order effects (difference in the magnitude of conflict effects following conflict vs. following non-conflict trials) further confirmed that (high-level) location-related and (low-level) prime-related control processes act independently, in line with the assumption that they are mediated by different neural systems ${ }^{2}$.

Employing the same hybrid prime-Simon task, the present study aimed to compare young adults' performance with that of normal healthy older adults. For young adults, we expected to replicate the earlier pattern of no interaction between the Simon effect and the NCE, that is, similar NCEs for congruent vs. incongruent Simon trials. For older adults, on the basis of previous studies listed above, we expected significantly (and disproportionately) greater Simon effects in terms of RTs, though not errors. Predictions regarding older adults' NCEs are less clear. On the one hand, based on previous studies, we did not expect NCEs in older adults for the primetarget interval used in the experiment $(150 \mathrm{~ms})$. On the other hand, there were two reasons for supposing that NCEs might appear, at least for incongruent trials. First, the additional delay to responses due to the Simon effect could be sufficient to compensate for the older adults' delayed onset of the NCE. Second, as already noted, there is evidence that inhibitory effects that do not interact in young adults may nevertheless interact in older adults (e.g., Germain and Collette, 2008), consistent with the dedifferentiation hypothesis of aging (e.g., Li et al., 2004). To disentangle these two possibilities, Schlaghecken, Birak, and Maylor's (in press) time course analysis was applied to the priming effects in the present study, separately for congruent and incongruent Simon trials.

\section{MATERIALS AND METHODS PARTICIPANTS}

There were two groups of participants, young and older, aged 18-30 and 63-82 years, respectively. Young participants $(n=23)$ were mostly students studying at the University of Warwick who took part either for course credit or for payment of $\mathfrak{E} 6$. Older participants $(n=28)$ were members of a volunteer panel who had been recruited through local newspapers and advertisements to join the Warwick Age Study. They were paid $£ 10$ to cover their travel expenses.

Background cognitive measures were collected from all but one of the young participants immediately after they had completed the experiment, whereas this information was already available for all older

${ }^{2}$ Note that if high-level and low-level conflict come from the same domain (e.g., prime shape and flanker shape in a hybrid prime-flanker task), interactions can be observed (see Boy et al., 2010). 
participants from an earlier testing session. Overall means are presented in Table 1; these do not include three young participants and one older participant who were excluded because of error rates greater than $15 \%$ in the main experiment. As an estimate of crystallized intelligence, vocabulary was assessed by the multiple choice section of the Mill Hill vocabulary test (Raven et al., 1988) in which participants are required to select the best synonym for a target word from a set of six alternatives. Speed of information processing was assessed by the Digit Symbol Substitution test from the Wechsler Adult Intelligence Scale-Revised (Wechsler, 1981).Visual acuity was assessed (with glasses if worn) at the beginning of the experimental session using the Near Vision Test Card (Schneider, 2002). All participants had normal or corrected-to-normal vision according to self-report. The statistical comparisons in Table 1 show the pattern expected from the aging literature (e.g., Salthouse, 1991; Baltes and Lindenberger, 1997; Schneider and Pichora-Fuller, 2000) of better vocabulary but reduced speed and poorer visual acuity in the older group than in the young group.

\section{STIMULI AND APPARATUS}

Stimuli were presented on a $17^{\prime \prime}$ computer screen in black on a white background. Primes and targets were left- and right-pointing double arrows $(<<$ and $>>)$, subtending a visual angle of $2.0^{\circ} \times 0.9^{\circ}$ at a viewing distance of approximately $1 \mathrm{~m}$. Primes were followed by a backward-masking stimulus to reduce prime visibility. These masks were constructed from a virtual $8 \times 6$ grid $\left(2.6^{\circ} \times 1.4^{\circ}\right)$, randomly filled with overlapping horizontal, vertical, and oblique lines of different lengths $\left(0.1^{\circ}\right.$ to $1.0^{\circ}$; width $\left.0.1^{\circ}\right)$. A new mask was constructed on each trial to avoid perceptual learning of the mask and correspondingly increased prime identification (see Schlaghecken et al., 2008). Primes and masks appeared in the center of the screen, whereas targets appeared $5.7^{\circ}$ to the left or right (measured center-to-center; see Figure 1A). Participants were seated in a comfortable chair in a dimly lit, sound attenuated chamber, with response buttons mounted on adjustable armrests under their left and right index fingers.

Table 1 | Numbers and background details (means and SDs) of young and older participants, and results of comparisons between age groups.

\begin{tabular}{llll}
\hline Variable & Young & Older & \\
\hline$N^{\prime}$ & $20 / 23$ & $27 / 28$ & \\
Males;Females & $7 ; 13$ & $10 ; 17$ & \\
\hline & $\boldsymbol{M}(\mathbf{S D})$ & $\boldsymbol{M}(\mathbf{S D})$ & Comparison \\
& & & \\
\hline Age (years) & $20.5(3.3)$ & $70.3(4.9)$ & - \\
Vocabulary & & $24.1(4.4)$ & $t(44)=-5.56, p<0.001$ \\
Speed $^{3}$ & $17.1(3.9)$ & $52.6(11.0)$ & $t(44)=6.95, p<0.001$ \\
Visual acuity $^{4}$ & $74.6(9.9)$ & $5.2(1.3)$ & $t(45)=3.79, p<0.001$ \\
\hline
\end{tabular}

${ }^{1}$ Number of participants whose data were included in the analyses/number of people tested.

${ }^{2}$ Vocabulary from the multiple choice section of the Mill Hill vocabulary test (Raven et al., 1988); maximum score = 33; data missing for one young participant. ${ }^{3}$ Processing speed based on the Digit Symbol Substitution test (Wechsler, 1981); data missing for one young participant.

${ }^{4} V$ isual acuity as measured by the number of lines read correctly from the Near Vision Test Card (Schneider, 2002) viewed at a distance of $16^{\prime \prime}$ whilst wearing corrective glasses, with scores ranging from 1 (16/160 - lowest acuity) to 9 (16/16 - highest acuity).

\section{PROCEDURE}

During experimental sessions of approximately $1 \mathrm{~h}$, participants carried out two versions of the experiment, one with masked primes (as in Figure 1) and the other with non-masked primes where an empty rectangular frame replaced the mask. The order of these two conditions was counterbalanced across participants in both age groups; only the masked prime condition is reported here.

The masked prime experiment comprised six blocks of 72 trials each, preceded by one practice block of 32 trials. At the end of each block, there was a short rest period of at least $20 \mathrm{~s}$, after which participants could initiate the next block. As illustrated in Figure 1A, trials began with a central fixation dot $\left(0.1^{\circ} \times 0.1^{\circ}\right)$ presented for $250 \mathrm{~ms}$, followed by a $650-\mathrm{ms}$ blank screen. Then a prime was presented for $33 \mathrm{~ms}^{3}$, which was replaced immediately by a $100-\mathrm{ms}$ mask. Following a blank screen for $50 \mathrm{~ms}$, the target was presented for $100 \mathrm{~ms}$. Participants were instructed to maintain central eye fixation throughout, and to respond as quickly and accurately as possible to the direction of the target arrows (i.e., a left-hand keypress to arrows pointing to the left, and a right-hand key-press to arrows pointing to the right), regardless of the location on the screen of the target arrows. Finally, the intertrial interval between target offset and the next fixation dot was $1300 \mathrm{~ms}$.

As shown in Figure 1B, trials were termed "compatible" when prime and target arrows pointed in the same direction and "incompatible" when they pointed in opposite directions. Also, trials where target direction and target location corresponded (e.g., left-pointing arrows appearing on the left of the screen) were termed "congruent" trials, whereas trials where direction and location were in conflict were termed "incongruent" trials. All trials within each block of 72 (2 prime compatibility levels $\times 2$ target congruency levels $\times 2$ responses) were presented randomly and with equal probability.

\section{DATA ANALYSES}

Repeated measures analyses of variance (ANOVAs) were performed on mean correct RTs and percentage error rates, combined across leftand right-hand responses. ANOVAs were initially conducted with task order included as a between-subjects factor (two levels: masked condition before vs. after non-masked condition) but in neither case was there any effect of order or any interactions involving order. Therefore the reported ANOVAs were conducted with age group as the only between-subject factor (two levels: young vs. older), and prime compatibility (two levels: compatible vs. incompatible) and target congruency (two levels: congruent vs. incongruent) as within-subject factors.

Additional analyses were conducted following the procedure outlined by Schlaghecken, Birak, and Maylor (in press), which allowed us to trace the development of priming effects over time by calculating the magnitude of priming effects separately for each of a series of RT latency bins. In short, we (i) calculated mean correct RTs for each of the eight conditions ( 2 prime compatibility $\times 2$ target congruency $\times 2$ response hands) for each participant and each of the six experimental blocks individually; (ii) replaced any

\footnotetext{
${ }^{3}$ In a staircase-based prime identification task (Schlaghecken, Birak, and Maylor, in press), mean prime identification thresholds did not significantly differ between young and older adults with similar background characteristics to the present participants. Moreover, both young and older thresholds significantly exceeded $33 \mathrm{~ms}$, suggesting that the masked primes in the present set-up were equally below threshold and subjectively subliminal for young and older adults.
} 

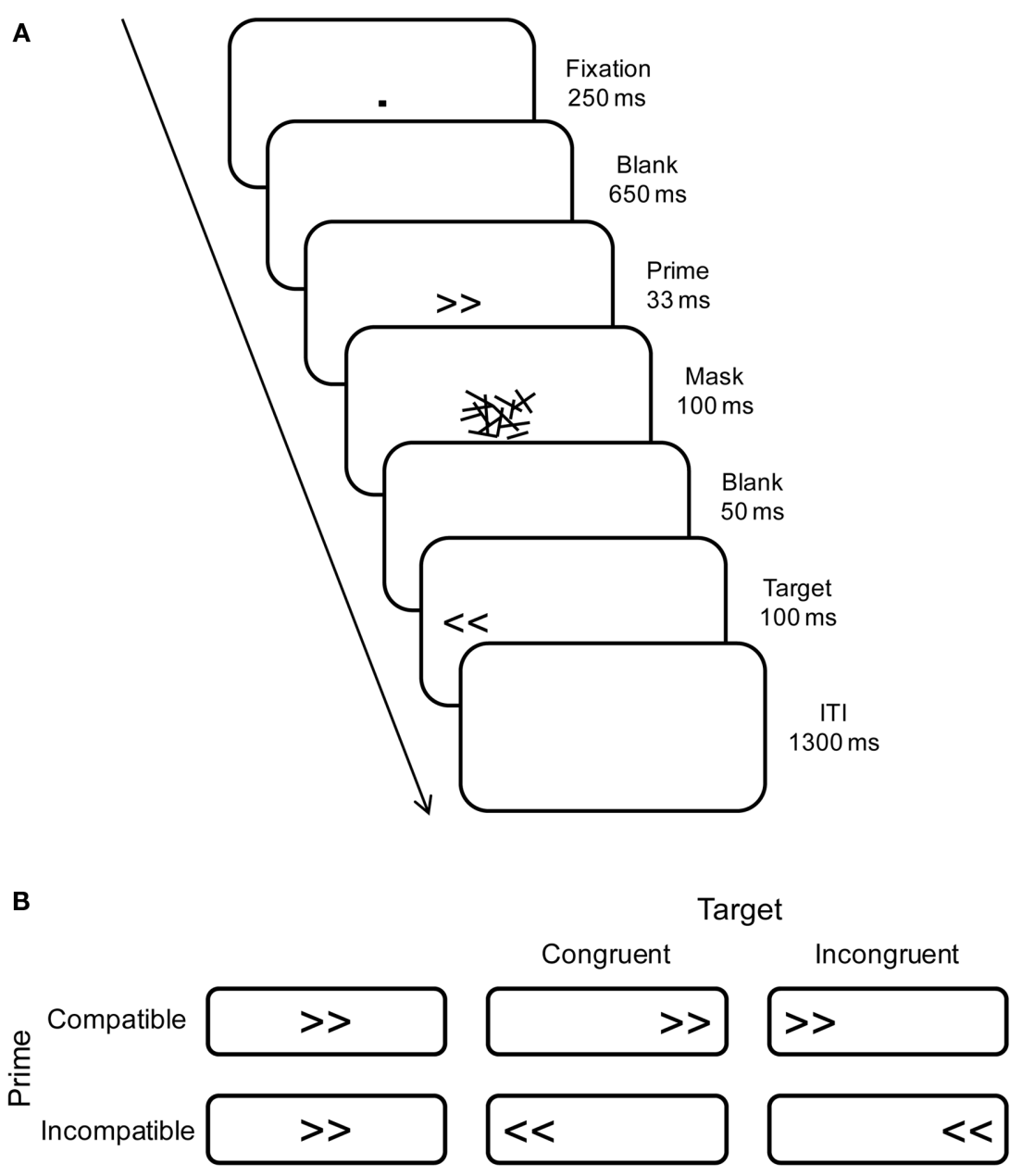

FIGURE 1 | (A) Schematic illustration of trials showing an example of an incompatible-prime congruent-target trial. (B) Summary of trials showing the four experimental conditions for right-pointing primes only (compatible/incompatible primes $\times$ congruent/incongruent targets).

error responses within a given block with the corresponding mean RT; (iii) rank-ordered RTs separately for each participant and each prime compatibility $\mathrm{x}$ target congruency condition; (iv) divided each RT distribution into nine latency bins of 12 trials each; (v) computed the mean latency of each bin by averaging across its compatible and incompatible RTs; and (vi) computed the priming effect within each bin by subtracting its mean compatible from its mean incompatible RT. Data from 1 older participant could not be analyzed because of insufficient numbers of correct responses in 1 (out of 8) conditions, leaving 20 young and 26 older participants. Latency bin 9 was excluded from the analysis because the data were more variable and therefore less reliable than bins 1-8 due to occasional slow outliers (the general pattern of results was the same with and without bin 9).

For statistical analyses, the following parameters were extracted for each participant, separately for congruent and incongruent trials: NCE peak latency (i.e., the latency bin containing the most negative priming effect), NCE peak amplitude (the magnitude of the priming effect within this bin), and priming slope (the slope of the linear regression function fitted through the priming effects). For each of these, repeated measures ANOVAs were conducted with the between-subject factor age (young, older), and the within-subject factor congruency (congruent, incongruent), and follow-up analyses were conducted in the form of paired $t$-tests, comparing effects on congruent and on incongruent trials separately within each age group.

\section{RESULTS}

\section{STANDARD ANALYSIS}

As shown by the means in Figure 2, and confirmed by the ANOVA results in Table 2, young adults were faster (by $150 \mathrm{~ms}$ ), but no less accurate, than older adults. Responses were faster and more accurate on incompatible than on compatible trials (NCE), and faster and more accurate on congruent than on incongruent trials (Simon effect). The NCE was larger in young than in older adults, both in terms of RTs and errors, whereas the Simon effect was larger in older than in young adults, at least in terms of RTs ${ }^{4}$. The NCE was less evident on congruent than on incongruent trials for both RTs and errors, although for errors this may be attributable to a floor effect (i.e., almost no errors) for congruent trials. Finally, the

${ }^{4}$ The marginal interaction for errors disappeared altogether with the removal of one young participant with an outlying error rate for compatible-incongruent trials. 


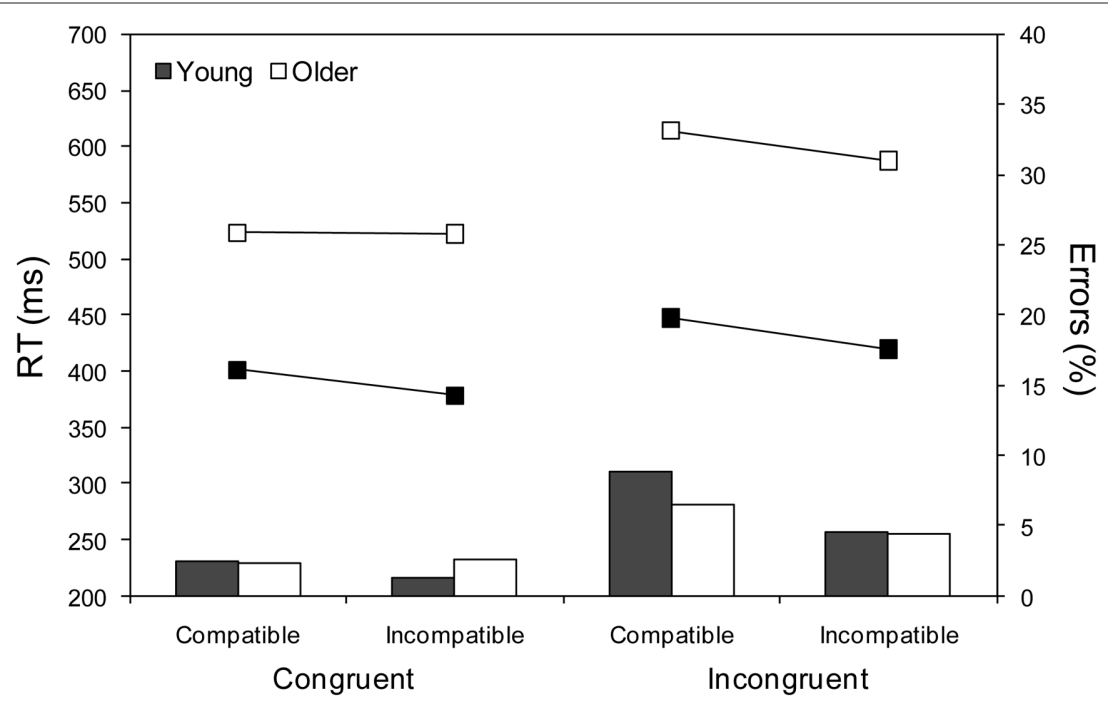

FIGURE 2 | Mean correct response times (RTs) in ms (lines) and percentage error rates (bars) for young and older adults as a function of prime type (compatible and incompatible) and target location (congruent and incongruent).

Table 2 | Summary of ANOVAs on mean correct response times (RTs) in $\mathrm{ms}$ and percentage error rates with age group (young vs. older), prime compatibility (compatible vs. incompatible), and target congruency (congruent vs. incongruent) as between-, within- and within-subject factors, respectively.

\begin{tabular}{|c|c|c|c|c|c|c|}
\hline \multirow{2}{*}{ Effect } & \multicolumn{3}{|c|}{ RTs } & \multicolumn{3}{|c|}{ Errors } \\
\hline & $F(1,45)$ & MSE & $p<$ & $F(1,45)$ & MSE & $p<$ \\
\hline Age & 86.36 & 11961.92 & 0.001 & 0.13 & 34.67 & n.s. \\
\hline PComp & 96.01 & 184.65 & 0.001 & 20.94 & 7.27 & 0.001 \\
\hline TCong & 195.26 & 869.10 & 0.001 & 62.64 & 11.37 & 0.001 \\
\hline Age $\times$ PComp & 7.83 & 184.65 & 0.01 & 5.25 & 7.27 & 0.03 \\
\hline Age ×TCong & 15.35 & 869.10 & 0.001 & 3.81 & 11.37 & 0.06 \\
\hline PComp $\times$ TCong & 57.93 & 47.72 & 0.001 & 16.25 & 5.51 & 0.001 \\
\hline Age $\times$ PComp $\times$ TCong & 24.96 & 47.72 & 0.001 & 0.27 & 5.51 & n.s. \\
\hline
\end{tabular}

Note: PComp = prime compatibility; TCong = target congruency.

difference in the NCE between congruent and incongruent trials for RTs was much greater for older than for young adults. This can be seen more clearly in Figure 3 where RT priming effects (incompatible - compatible) are plotted for congruent and incongruent trials. Thus whereas the NCE was similar for young adults across the two conditions, $t(19)=1.60, p>0.1$, it was markedly different for older adults, $t(26)=10.30, p<0.001$, from absent on congruent trials to normal on incongruent trials.

To examine whether the age-related increase in the Simon effect for RTs (from $44 \mathrm{~ms}$ in young to $78 \mathrm{~ms}$ in older participants) was greater than would be expected on the basis of generalized/proportional slowing, two different procedures were adopted. First, a further ANOVA was conducted after applying a logarithmic transformation to the data (cf. Van der Lubbe and Verleger, 2002). All the main effects and interactions were highly significant, $p<0.001$,

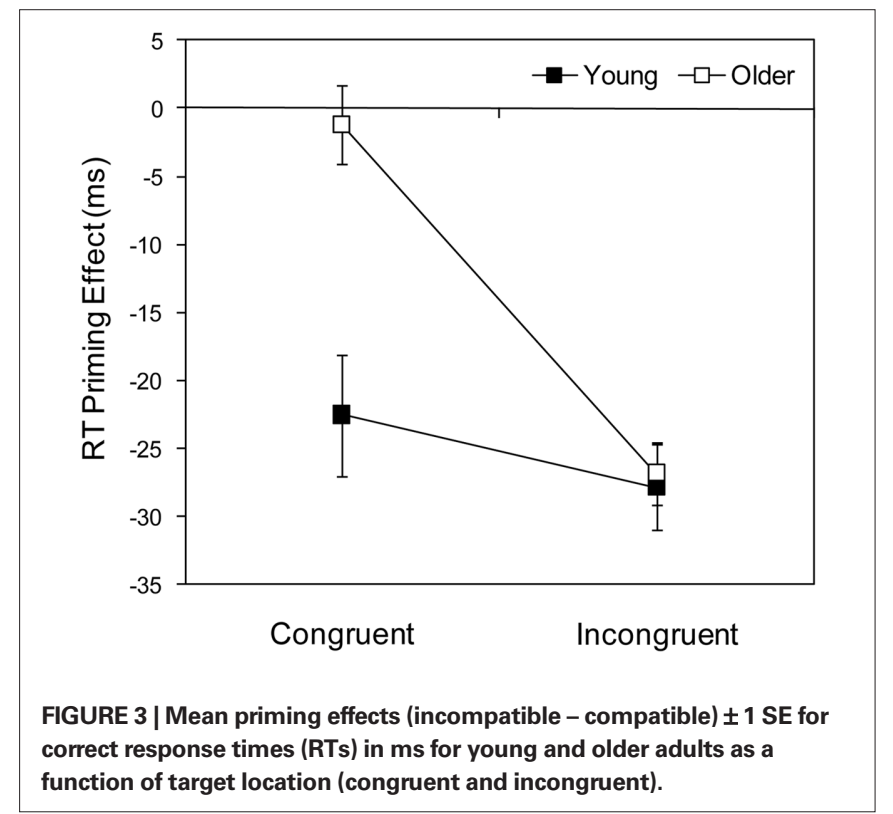

except for the age group $\mathrm{x}$ target congruency interaction, which was marginally significant, $F(1,45)=3.46, \mathrm{MSE}<0.001, p=0.069$. Second, standardized $z$ scores were calculated for congruent and incongruent conditions by taking each person's condition mean, subtracting their overall mean, and then dividing by the SD of the condition mean (cf. Castel et al., 2007; see also Faust et al., 1999). The mean difference in $z$ scores between congruent and incongruent conditions (Simon effect) was $0.65(\mathrm{SD}=0.31)$ for young adults, and $0.85(\mathrm{SD}=0.45)$ for older adults, $t(43.3)=-1.82, p=0.076$, resulting in a Cohen's $d$ value of 0.51 and therefore a medium effect size. Thus both procedures provide marginal support (significant on 1-tailed tests) for the suggestion that the age-related increase in the Simon effect is disproportionate. 
The absence of an interaction between the NCE and the Simon effect in young adults perfectly replicates the results of Schlaghecken, Refaat, and Maylor (in press). For older adults, the absence of an NCE for congruent trials is consistent with Schlaghecken, Birak, and Maylor's (in press, Figure 2) findings. The novel feature of the present results is the appearance of a highly reliable NCE in older adults for incongruent trials. As noted above, detailed time course analysis in our earlier study revealed NCEs in the majority of older adults, of the same magnitude as those of young adults, but substantially delayed. It seems plausible that in the present experiment, the Simon effect delayed responses in older participants sufficiently for the NCE to develop.

\section{TIME COURSE ANALYSIS}

Figure 4 shows mean RT priming effects plotted against mean RTs for the first eight latency bins, separately for congruent and incongruent trials. For young adults, NCEs show a mildly U-shaped function with similar peak amplitudes and peak latencies. For older adults, in contrast, NCEs show marked negative-going slopes, and peak amplitude as well as peak latency are larger on incongruent than on congruent trials. In the congruent condition, older adults' priming effects are initially positive (PCEs) and turn into subsequent NCEs with increasing RT, hence the absence of an overall priming effect (Figure 3).

For each participant and each condition separately, the significance of priming effects within each latency bin was determined by comparing compatible and incompatible RTs with an unpaired $t$-test, using a Bonferroni-corrected significance criterion. For congruent trials, a significant NCE occurred in at least one latency bin for $18 / 20$ young participants, but only for 14/26 older participants, $\chi^{2}(1)=6.98, p<0.01$. For incongruent trials, a significant NCE occurred in at least one latency bin for 20/20 young and 25/26 older participants. (One older participant failed to produce a significant NCE in any latency bin for either congruent or incongruent trials.)

All three time course parameters showed significant age group $\mathrm{x}$ target congruency interactions. First, whereas there was no statistically significant difference between congruent and incongruent NCE peak latency bins in young adults [congruent: $M=4.95, \mathrm{SD}=2.70$, incongruent: $M=3.85, \mathrm{SD}=2.66$; $t(19)=1.23, p>0.2$ ], in older adults the NCE peak was significantly delayed on incongruent relative to congruent trials [congruent: $M=5.96, \mathrm{SD}=2.38$, incongruent: $M=7.15, \mathrm{SD}=1.97$; $t(25)=2.43, p=0.022]^{5}, F(1,44)=5.71, \mathrm{MSE}=5.20, p=0.021$. Furthermore, the overall delay in NCE peak in older relative to young adults was reflected in a significant main effect of age, $F(1$, $44)=16.37, \mathrm{MSE}=6.43, p<0.001$. Second, in young adults, NCE peak amplitude ( $\mathrm{ms}$ ) was unaffected by target congruency [congruent: $M=43.4, \mathrm{SD}=23.3$, incongruent: $M=40.0, \mathrm{SD}=17.6$; $t(19)<1$ ], whereas in older adults, NCE peak amplitude was significantly enhanced on incongruent relative to congruent trials [congruent: $M=27.2, \mathrm{SD}=34.6$, incongruent: $M=52.1$, $\mathrm{SD}=23.8 ; t(25)=4.70, p<0.001], F(1,44)=16.69, \mathrm{MSE}=271.66$, $p<0.001$. Third, and most importantly, the slope of priming effects was unaffected by target congruency in young participants [congruent: $M=-0.04, \mathrm{SD}=0.26$, incongruent: $M=0.04$, $\mathrm{SD}=0.15 ; t(19)=1.10, p>0.28]$, but was significantly more negative on incongruent than congruent trials in older adults [congruent: $M=-0.17, \mathrm{SD}=0.34$, incongruent: $M=-0.28, \mathrm{SD}=0.42$; $t(25)=2.67, p=0.013], F(1,44)=6.10, \mathrm{MSE}=0.03, p=0.017$. Also, there was a significant main effect of age, $F(1,44)=6.53$, MSE $=0.17, p=0.014$, as priming effect slopes were generally more negative in older than in young participants.

Finally, the Simon effect is visible in Figure 4 as a right-shift of incongruent relative to congruent curves. Note that the disproportionate increase in Simon effect magnitude in older adults becomes apparent in the fact that for young adults, the first bin of the incongruent curve coincides with the third bin of the congruent curve, whereas for older adults, it coincides with the fourth. An

${ }^{5}$ Note that these values do not exactly correspond with Figure 4 because of between-participant variability in the shape of the functions - hence, the overall latency bin for the most negative priming effect calculated by taking each participant's peak bin and then averaging across participants is not necessarily the same as that obtained from averaging priming effects across participants and then taking the NCE peak latency bin.

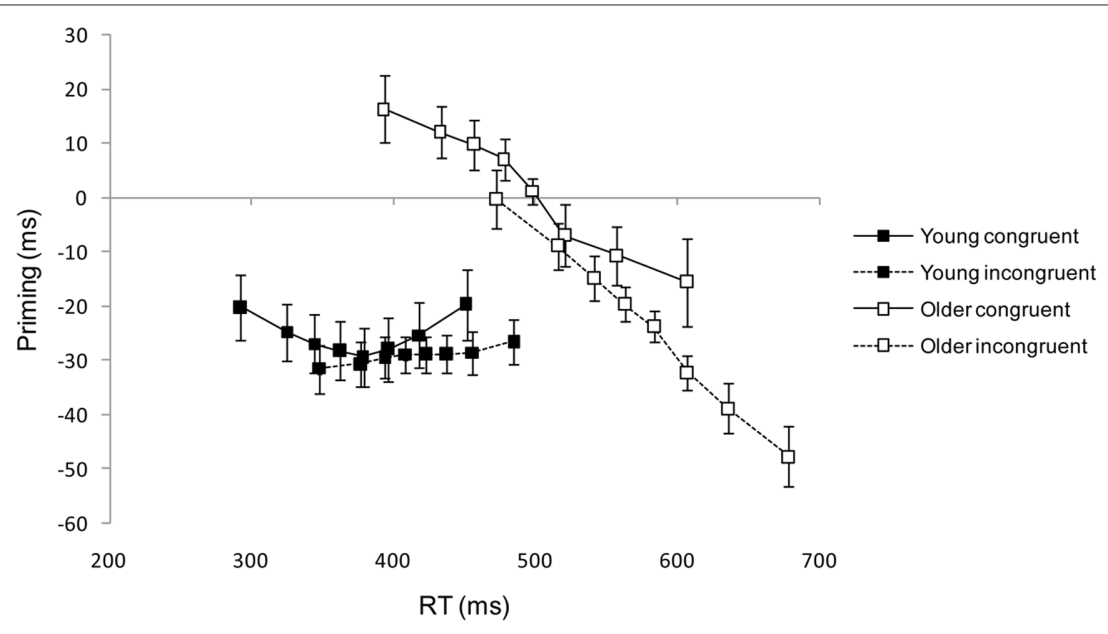

FIGURE 4 | Mean priming effects (incompatible - compatible RT) \pm 1 SE in ms plotted as a function of mean response times (RTs) for each of eight RT latency bins from the time course analysis (see text for details). Data are plotted separately for young and older adults and for congruent and incongruent trials. 
additional feature, perhaps less readily apparent from Figure 4, is the changing size of the Simon effect across the RT distribution. For young adults, there was an almost linear decrease in the Simon effect from 55 to $34 \mathrm{~ms}$ across bins $1-8$, which is consistent with previous studies (e.g., De Jong et al., 1994; Pratte et al., 2010). Some suggest that this decrease reflects passive decay over time in the activation of the response code corresponding to the location of the stimulus (Hommel, 1994); others attribute it to active suppression (Ridderinkhof, 2002). Previous studies are less consistent with respect to older adults: Kubo-Kawai and Kawai (2010), Proctor et al. (2005), and Van der Lubbe and Verleger (2002) found the same decreasing pattern in older adults as in young adults, whereas both Castel et al. (2007) and Juncos-Rabadán et al. (2008) found a decrease in young adults but an increase in older adults. The present older adults showed a small monotonic increase in the Simon effect from 79 to $86 \mathrm{~ms}$ across bins $1-6$, followed by a monotonic decrease to $72 \mathrm{~ms}$ by bin 8 . Thus there is greater support here for Castel et al.'s (2007) conclusion of a more sustained influence of the irrelevant stimulus location across the RT distribution in older than in young adults.

\section{DISCUSSION}

The present study employed a hybrid procedure combining the Simon task with the masked prime task to investigate both highand low-level inhibitory control processes and their interactions in young and older adults. Results can be summarized as follows:

(1) Consistent with previous findings (e.g., Van der Lubbe and Verleger, 2002; Castel et al., 2007), the Simon effect was significantly larger in older than in young adults. Importantly, this age difference was not entirely accounted for by general slowing, suggesting a disproportionate impairment in highlevel consciously controlled inhibitory processes in old age (cf. Hasher and Zacks, 1988).

(2) Standard RT analysis (Figures 2 and 3) showed reliable NCEs in young adults for both congruent and incongruent Simon trials, whereas older adults showed a reliable NCE for incongruent trials only. The aging pattern for target-congruent trials (NCE in young but not in older adults) replicates results from an earlier masked prime study with centrally presented targets (Schlaghecken and Maylor, 2005), thus supporting the conclusion that low-level automatically controlled inhibitory processes are not always spared in normal aging, contrary to some accounts (e.g., Andres et al., 2008; Collette et al., 2009).

(3) However, the fine-grained time course analysis (Figure 4) confirmed Schlaghecken, Birak, and Maylor's (in press) more recent demonstration that the NCE is not completely absent in older adults, but rather is disproportionately delayed (i.e., delayed beyond overall slowing, as evidenced by the significant difference in NCE peak latency bins between young and older adults).

(4) Importantly, the time course analysis also confirmed that in older but not in young adults, priming effects and Simon effects interact. Not only was the incongruent-trial NCE peak larger than the congruent-trial NCE peak in older but not in young adults (a result already seen in the standard analysis), but it was also significantly delayed in older but not in young adults. Perhaps the most relevant finding in this context is that in older but not in young adults, the slope of priming effects was significantly steeper on incongruent than on congruent trials, suggesting more powerful inhibition on these trials (e.g., Ridderinkhof, 2002).

Taken together, these results suggest the following scenario. In young adults, the inhibitory control of motor activation triggered by the shape of a non-consciously perceived (masked) prime acts independently of the inhibitory control of motor activation triggered by the location of a consciously perceived target, as evidenced by the absence of any prime compatibility $\mathrm{x}$ target congruency interaction. This result replicates Schlaghecken, Refaat, and Maylor's (in press) findings, and is consistent with the conclusion that in young adults, high- and low-level inhibitory control processes are mediated by separate and independent neural systems. In older adults, in contrast, prime compatibility effects and target congruency effects interact, suggesting that they are generated by processes that share neural resources.

It is worth noting that this interaction cannot be explained fully by general slowing. Older adults produce NCEs of the same magnitude as young adults, but at a much increased latency (Schlaghecken, Birak, and Maylor, in press). Therefore, one might argue that in the present experiment, older adults' incongruenttrial NCEs were larger than their congruent-trial NCEs because on incongruent trials, responses were slower than on congruent trials (Simon effect), allowing inhibition more time to develop. However, this interpretation is contrary to two aspects of the data. First, in Experiment 1 of Schlaghecken, Birak, and Maylor's (in press) study, primes and targets were separated by intervals of up to $450 \mathrm{~ms}$, allowing us to investigate prime-response latencies that were much longer than those observed in the present study. The NCE reached its peak magnitude for responses occurring on average $548 \mathrm{~ms}$ after mask onset for young adults and $765 \mathrm{~ms}$ after mask onset for older adults. For a mask-target stimulus onset asynchrony of $150 \mathrm{~ms}$ (as employed in the present study), these would correspond to young and older RTs of 398 and 615 ms, respectively. Comparing these values with Figure 4, the present young adults' NCEs similarly peaked at RTs around $400 \mathrm{~ms}$, for both congruent and incongruent Simon trials. However, for older adults, the NCE for incongruent trials in particular had not reached its peak magnitude by $615 \mathrm{~ms}$ but was still increasing at longer RTs. This indicates that longer response latencies as such are not sufficient to explain older adults' large NCE on incongruent trials. Second, this conclusion is further supported by the finding that in older participants, the slope of priming effects (i.e., the rate of inhibition) was significantly more negative for incongruent than for congruent trials, suggesting that on incongruent trials, more - or more effective-inhibitory control was available than on congruent trials (e.g., Ridderinkhof, 2002).

The present findings of additive inhibitory effects in young but interactive effects in older adults correspond closely with those of Germain and Collette (2008). These authors used tasks involving "perceptual" conflict (e.g., respond with a left/right key-press to a left-/right-pointing arrow regardless of its left/right location on the screen), tasks involving "motor" conflict (e.g., respond with 
a left/right key-press to a right-/left-pointing arrow at fixation), and tasks involving both (e.g., respond with a left/right key-press to a right-/left-pointing arrow regardless of its left/right location on the screen). Their results suggested that perceptual and motor inhibitory processes draw on separate cognitive resources in young adults, but on shared cognitive resources in older adults, which the authors interpreted in terms of dedifferentiation in old age.

We propose a similar model for the present results: in young adults, inhibition of motor tendencies triggered by masked primes and inhibition of motor tendencies triggered by taskirrelevant target location are separate and independent processes, whereas in older adults, they rely on shared cognitive or neural resources. Moreover, the pattern of results suggests a specific direction of these dedifferentiation effects, namely, a shift toward high-level and away from low-level, automatic control processes. The evidence for this is twofold. First, in the present study as well as in Schlaghecken, Birak, and Maylor (in press), older adults' NCEs were disproportionally delayed relative to young participants' NCEs (i.e., delayed more than can be accounted for by general slowing). One of the remarkable features of the NCE in young adults is that it occurs early: a prime-target interval of only $150 \mathrm{~ms}$ is sufficient for this effect to occur. In contrast, reversed priming effects with non-masked primes are only observed with intervals of about 800-1200 ms (e.g., Machado et al., 2007; Jaśkowski, 2008a), at least as long as non-masked primes are not immediately followed by potentially task-relevant, target-like distractors, which might actively encourage the activation of the prime-opposite response (e.g., Jaśkowski, 2008b). These differences in timing are consistent with the notion that the NCE with masked primes occurs at early, lowlevel stages of the perceptuo-motor processing stream (e.g., Aron et al., 2003), whereas inhibitory control of consciously perceived irrelevant information involves higher, presumably prefrontal processing stages that take noticeably longer to become activated. If this reasoning is correct, then the disproportionate delay of

\section{REFERENCES}

Andres, P., Guerrini, C., Phillips, L. H., and Perfect, T. J. (2008). Differential effects of aging on executive and automatic inhibition. Dev. Neuropsychol. 33, 101-123.

Aron, A., Schlaghecken, F., Fletcher, P., Bullmore, E., Eimer, M., Barker, R., Sahakian, B., and Robbins, T. (2003). Inhibition of subliminally primed responses is mediated by the caudate and thalamus: evidence from fMRI and Huntington's disease. Brain 126, 713-723.

Baltes, P. B., and Lindenberger, U. (1997). Emergence of a powerful connection between sensory and cognitive functions across the adult life span: a new window to the study of cognitive aging? Psychol. Aging 12, 12-21.

Bialystok, E., Craik, F. I. M., Klein, R., and Viswanathan, M. (2004). Bilingualism, aging, and cognitive control: evidence from the Simon task. Psychol. Aging 19, 290-303.
Boy, F., Clarke, K., and Sumner, P. (2008). Mask stimulus triggers inhibition in subliminal visuo-motor priming. Exp. Brain Res. 190, 111-116.

Boy, F., Husain, M., and Sumner, P. (2010). Unconscious inhibition separates two forms of cognitive control. Proc. Natl. Acad. Sci. 107, 11134-11139.

Burke, D. M. (1997). Language, aging, and inhibitory deficits: evaluation of a theory. J. Gerontol. Psychol. Sci. 52B, P254-P264.

Castel, A. D., Balota, D. A., Hutchison, K. A., Logan, J. M., and Yap, M. J. (2007). Spatial attention and response control in healthy younger and older adults and individuals with Alzheimer's disease: evidence for disproportionate selection impairments in the Simon task. Neuropsychology 21, 170-182.

Cerella, J. (1990). "Aging and informationprocessing rate," in Handbook of the Psychology of Aging, 3rd Edn, eds J. E. Birren and K. W. Schaie (San Diego, CA: Academic Press), 201-221.
NCEs in older participants suggests that they cannot successfully generate this effect within early, low-level, and automatic stages, but instead require intervention of higher, prefrontally mediated inhibitory control for the NCE to occur.

The second line of evidence for a de-automatization of inhibitory control in old age comes from the fact that older participants' NCEs were selectively enhanced on incongruent relative to congruent trials. On incongruent-location trials, high-level motor inhibition is required to overcome the incorrect response tendency; on congruentlocation trials, this is not the case. Therefore the finding that older participants' NCEs were larger, showed a steeper slope, and occurred even later on incongruent than on congruent trials is consistent with the idea that on incongruent trials, weak low-level inhibition received a "boost" from - or was substituted by - high-level control activity.

Evidence for age-related de-automatization of motor control has been observed in other paradigms unrelated to the masked prime task. Specifically, it would appear that with increasing age, motor control during simple automatic tasks shifts from highly efficient and automatic low-level processes to less efficient and less automatic high-level processes, with a correspondingly greater recruitment of prefrontal and parietal cortical regions (see Schlaghecken, Birak, and Maylor, in press, for an overview). In view of marked age-related decline particularly in prefrontal brain areas (see Dennis and Cabeza, 2008) such a pattern of compensation seems less than ideal. Nonetheless, the suggestion that older adults may rely more than young adults on high-level (prefrontally mediated) inhibition in the masked prime task fits well with the present pattern of interacting inhibitory control processes in older adults, and with the wider evidence. However, neuroimaging studies would be required to confirm such speculations.

\section{ACKNOWLEDGMENTS}

This research was supported by a grant to Elizabeth Ann Maylor and Friederike Schlaghecken from the Economic and Social Research Council of Great Britain (RES-000-22-2687).

Collette, F., Germain, S., Hogge, M., and Van der Linden, M. (2009). Inhibitory control of memory in normal ageing: dissociation between impaired intentional and preserved unintentional processes. Memory 17, 104-122.

De Jong, R., Liang, C.-C., and Lauber, E. (1994). Conditional and unconditional automaticity: a dualprocess model of effects of spatial stimulus-response correspondence. J. Exp. Psychol. Hum. Percept. Perform. 20, 731-750.

Dennis, N. A., and Cabeza, R. (2008). "Neuroimaging of healthy cognitive aging," in The Handbook of Aging and Cognition, 3rd Edn, eds F. I. M. Craik and T. A. Salthouse (New York: Psychology Press), 1-54.

Duchek, J. M., Balota, D. A., Tse, C.-S., Holtzman, D. M., Fagan, A. M., and Goate, A. M. (2009). The utility of intraindividual variability in selective attention tasks as an early marker for
Alzheimer's disease. Neuropsychology 23, 746-758.

Eimer, M., and Schlaghecken, F. (1998). Effects of masked stimuli on motor activation: behavioral and electrophysiological evidence. J. Exp. Psychol. Hum. Percept. Perform. 24, 1737-1747.

Eimer, M., and Schlaghecken, F. (2002). Links between conscious awareness and response inhibition: evidence from masked priming. Psychon. Bull. Rev. 9, 514-520.

Faust, M. E., Balota, D. A., Spieler, D. H., and Ferraro, F. R. (1999). Individual differences in information-processing rate and amount: implications for group differences in response latency. Psychol. Bull. 125, 777-799.

Germain, S., and Collette, F. (2008). Dissociation of perceptual and motor inhibitory processes in young and elderly participants using the Simon task. J. Int. Neuropsychol. Soc. 14, 1014-1021. 
Guerreiro, M. J. S., Murphy, D. R., and Van Gerven, P. W. M. (2010). The role of sensory modality in agerelated distraction: a critical review and a renewed view. Psychol. Bull. 136, 975-1022.

Hasher, L., and Zacks, R. T. (1988). "Working memory, comprehension, and aging: a review and a new view," in The Psychology of Learning and Motivation, Vol. 22, ed. G. H. Bower (New York: Academic Press), 193-225.

Hasher, L., Zacks, R. T., and May, C. P. (1999). "Inhibitory control, circadian arousal, and age," in Attention and Performance XVII. Cognitive Regulation of Performance: Interaction of Theory and Application, eds D. Gopher and A. Koriat (Cambridge, MA: MIT Press), 653-675.

Hommel, B. (1994). Spontaneous decay of response-code activation. Psychol. Res. 56, 261-268.

Jaśkowski, P. (2008a). Conscious contributions to subliminal priming. Conscious. Cogn. 17, 72-83.

Jaśkowski, P. (2008b). The negative compatibility effect with nonmasking flankers: a case for mask-triggered inhibition hypothesis. Conscious. Cogn. 17, 765-777.

Juncos-Rabadán, O., Pereiro, A. X., and Facal, D. (2008). Cognitive interference and aging: insights from a spatial stimulus-response consistency task. Acta Psychol. 127, 237-246.

Kramer, A. F., Humphrey, D. G., Larish, J. F., Logan, G. D., and Strayer, D. L. (1994). Aging and inhibition: beyond a unitary view of inhibitory processing in attention. Psychol. Aging 9,491-512.

Kubo-Kawai, N., and Kawai, N. (2010). Elimination of the enhanced Simon effect for older adults in a three-choice situation: ageing and the Simon effect in a go/no-go Simon task. Q. J. Exp. Psychol. 63, 452-464.

Li, S.-C., Lindenberger, U., Hommel, B., Aschersleben, G., Prinz,W., and Baltes, P. B. (2004). Transformations in the couplings among intellectual abilities and constituent cognitive processes across the life span. Psychol. Sci. 15, 155-163.

Liu, X., Banich, M. T., Jacobson, B. L., and Tanabe, J. L. (2004). Common and distinct neural substrates of attentional control in an integrated Simon and spatial Stroop task as assessed by event-related fMRI. Neuroimage 22, 1097-1106.

Lu, C.-H., and Proctor, R. W. (1995). The influence of irrelevant location information on performance: a review of the Simon and spatial Stroop effects. Psychon. Bull. Rev. 2, 174-207.

Machado, L., Wyatt, N., Devine, A., and Knight, B. (2007). Action planning in the presence of distracting stimuli: an investigation into the time course of distractor effects. J. Exp. Psychol. Hum. Percept. Perform. 33, 1045-1061.

Maylor, E. A., Schlaghecken, F., and Watson, D. G. (2005). "Aging and inhibitory processes in memory, attentional, and motor tasks," in Cognitive Limitations in Aging and Psychopathology, eds R. W. Engle, G. Sedek, U. von Hecker, and D. N. McIntosh (New York: Cambridge University Press), 313-345.

McDowd, J. M. (1997). Inhibition in attention and aging. J. Gerontol. Psychol. Sci. 52B, P265-P273.

Myerson, J., Hale, S., Wagstaff, D., Poon, L. W., and Smith, G. A. (1990). The information-loss model: a mathematical theory of age-related cognitive slowing. Psychol. Rev. 97, 475-487.

Peterson, B. S., Kane, M. J., Alexander, G. M., Lacadie, C., Skudlarski, P., Leung, H.-C., May, J., and Gore, J. C. (2002). An event-related functional MRI study comparing interference effects in the Simon and Stroop tasks. Cogn. Brain Res. 13, 427-440.

Pratte, M. S., Rouder, J. N., Morey, R. D., and Feng, C. N. (2010). Exploring the differences in distributional properties between Stroop and Simon effects using delta plots. Atten. Percept. Psychophys. 72, 2013-2025.

Proctor, R. W., Pick, D. F., Vu, K.-P. L., and Anderson, R.E. (2005). The enhanced Simon effect for older adults is reduced when the irrelevant location information is conveyed by an accessory stimulus. Acta Psychol. 119, 21-40.

Proctor, R. W., and Vu, K.-P. L. (2006). Stimulus-Response Compatibility Principles. Boca Raton, FL: Taylor \& Francis.

Rabbitt, P. M. A. (1965). An age-decrement in the ability to ignore irrelevant information. J. Gerontol. 20, 233-237.

Raven, J. C., Raven, J., and Court, J. H. (1988). The Mill Hill Vocabulary Scale. London: H. K. Lewis Co. Ltd.

Raz, N. (2000). "Aging of the brain and its impact on cognitive performance: integration of structural and functional findings," in The Handbook of Aging and Cognition, 2nd Edn, eds F. I. M. Craik and T. A. Salthouse (Mahwah, NJ: Erlbaum), 1-90.

Ridderinkhof, K. R. (2002). "Activation and suppression in conflict tasks: empirical clarification through distributional analysis," in Common Mechanisms in Perception and Action. Attention and Performance, XIX, eds W. Prinz and B. Hommel (Oxford: Oxford University Press), 494-519.

Salthouse, T. A. (1991). Theoretical Perspectives on Cognitive Aging. Hillsdale, NJ: Erlbaum.
Salthouse, T. A. (1996). The processingspeed theory of adult age differences in cognition. Psychol. Rev. 103, 403-428.

Schlaghecken, F., Birak, K. S., and Maylor, E. A. (in press). Age-related deficits in low-level inhibitory motor control. Psychol. Aging.

Schlaghecken, F., Blagrove, E., and Maylor E. A. (2008). No difference between conscious and nonconscious visuomotor control: evidence from perceptual learning in the masked prime task. Conscious. Cogn. 17, 84-93.

Schlaghecken, F., and Eimer, M. (1997). The influence of subliminally presented primes on response preparation. $Z$. Psychol.Z. Angew. Psychol. 16, 166-175.

Schlaghecken, F., and Eimer, M. (2002). Motor activation with and without inhibition: evidence for a threshold mechanism in motor control. Percept. Psychophys. 64, 148-162.

Schlaghecken, F., and Eimer, M. (2006). Active masks and active inhibition: a comment on Lleras and Enns (2004) and on Verleger, Jaśkowski, Aydemir, van der Lubbe, and Groen (2004). J. Exp. Psychol. Gen. 135, 484-494.

Schlaghecken, F., Klapp, S. T., and Maylor E. A. (2009). Either or neither, but not both: locating the effects of masked primes. Proc. R. Soc. Lond. B 276 515-521.

Schlaghecken, F., and Maylor, E. A. (2005). Motor control in old age: evidence of impaired low-level inhibition. $J$. Gerontol. Psychol. Sci.60B, P158-P161.

Schlaghecken, F., Refaat, M., and Maylor, E. A. (in press). Multiple systems for cognitive control: evidence from a hybrid prime-Simon task. J. Exp. Psychol. Hum. Percept. Perform.

Schlaghecken, F., Rowley, L., Sembi, S., Simmons, R., and Whitcomb, D. (2007). The negative compatibility effect: a case for inhibition. Adv. Cogn. Psychol. 3, 227-240.

Schlaghecken, F., and Sisman, R. (2006). Low-level motor inhibition in children: evidence from the negative compatibility effect. Adv. Cogn. Psychol. 2, 7-19.

Schneider, B. A., and Pichora-Fuller, M. K. (2000). "Implications of perceptual deterioration for cognitive aging research," in The Handbook of Aging and Cognition, 2nd Edn, eds F. I. M. Craik and T. A. Salthouse (Mahwah, NJ: Erlbaum), 155-219.

Schneider, J. (2002). Near Vision Test Card. Available at: http://www.i-see.org/ block_letter_eye_chart.pdf [accessed November 16, 2007].

Seiss, E., and Praamstra, P. (2004). The basal ganglia and inhibitory mechanisms in response selection: evidence from subliminal priming of motor responses in Parkinson's disease. Brain $127,330-339$.
Seiss, E., and Praamstra, P. (2006). Timecourse of masked response priming and inhibition in Parkinson's disease. Neuropsychologia 44, 869-875.

Simon, J. R., and Pouraghabagher, A. R. (1978). The effect of aging on the stages of processing in a choice reaction time task. J. Gerontol.33,553-561.

Sumner, P. (2007). Negative and positive masked-priming - implications for motor inhibition. Adv. Cogn. Psychol. 3, 317-326.

Sumner, P., Nachev, P., Morris, P., Peters, A. M., Jackson, S. R., Kennard, C., and Husain, M. (2007). Human medial frontal cortex mediates unconscious inhibition of voluntary action. Neuron 54, 697-711.

Tse, C.-S., Balota, D. A., Yap, M. J., Duchek, J.M., and McCabe, D. P. (2010). Effects of healthy aging and early stage dementia of the Alzheimer's type on components of response time distributions in three attention tasks. Neuropsychology 24, 300-315.

Van der Lubbe, R. H. J., and Verleger, R. (2002). Aging and the Simon task. Psychophysiology 39, 100-110.

Wechsler, D. (1981). Manual for the Wechsler Adult Intelligence ScaleRevised. New York: Psychological Corporation.

West, R. L. (1996). An application of prefrontal cortex function theory to cognitive aging. Psychol. Bull. 120, 272-292.

Zacks, R. T., Hasher, L., and Li, K. Z. H. (2000). "Human memory," in The Handbook of Aging and Cognition, 2nd Edn, eds F. I. M. Craik and T. A. Salthouse (Mahwah, NJ: Erlbaum), 293-357.

Conflict of Interest Statement: The authors declare that the research was conducted in the absence of any commercial or financial relationships that could be construed as a potential conflict of interest.

Received: 03 May 2011; accepted: 06 June 2011; published online: 20 June 2011.

Citation: Maylor EA, Birak KS and Schlaghecken F (2011) Inhibitory motor control in old age: evidence for de-automatization? Front. Psychology 2:132. doi: 10.3389/fpsyg.2011.00132

This article was submitted to Frontiers in Cognition, a specialty of Frontiers in Psychology.

Copyright () 2011 Maylor, Birak and Schlaghecken. This is an open-access article subject to a non-exclusive license between the authors and Frontiers Media SA, which permits use, distribution and reproduction in other forums, provided the original authors and source are credited and other Frontiers conditions are complied with. 